Grażyna Strzelecka

\title{
Zum Gebrauch von Phrasen und Metaphern in der Wirtschaftssprache - eine Untersuchung mit fachsprachendidaktischen Aspekten
}

Grażyna Strzelecka: A szószerkezetek és a metaforák használata a gazdasági nyelvben - vizsgálat szaknyelvoktatási szempontból

\section{Összefoglaló}

A tanulmány a régebbi és az újabb gazdasági szaksajtóban elöforduló szószerkezetek és metaforák használatához füz néhány gondolatot. A példák a sajtócikkek pédamondatain keresztül kontextusba ágyazottan kerülnek bemutatásra. Számos idézet segítségével vizsgálom az adott időintervallumban közkedvelt, a gazdasági szaknyelvben vissza-visszatérö kifejezéseket. A témát nyelvtudományi és nyelvoktatási szemszögböl közelítem meg, mivel e specifikus szókapcsolatok ismerete mind a gazdasági szövegek feltárásához, mind a szakkifejezések megtanulásához hasznos lehet. Érintem azt a kérdést is, hogy a gazdasági szaknyelvben mely szóalkotási jelenségek járulnak hozzá újabb szakszavak kialakulásához, és ezáltal a gazdasági nyelv további fejlödéséhez. Ez a fejlődés újabb kihívások elé állitja az idegennyelv-oktatást, mivel a gazdasági szakkifejezések növekvő mértékben áramlanak át a köznyelvbe is.

Kulcsszavak: gazdasági nyelv, gazdasági sajtó, szószerkezet, metafora

\begin{abstract}
Der Vortrag stellt einige Gedanken zum Gebrauch beliebter Phrasen und Metaphern in der alten und neueren Wirtschaftspresse dar, die anhand von Satzbeispielen aus Presseartikeln im Kontext gezeigt werden. Mit Hilfe von mehreren Zitaten werden Ausdrücke der Wirtschaftssprache analysiert, die sich in dem erforschten Zeitfenster großer Beliebtheit erfreuen. Das Thema wird aus sprachwissenschaftlicher, aber auch aus sprachdidaktischer Sicht betrachtet, denn das Wissen um diese spezifischen Redensarten kann beim Erschließen von Wirtschaftstexten und beim Erlernen der Fachausdrücke der Wirtschaftssprache von Nutzen sein. Angesprochen wird auch die Frage, welche Wortbildungsphänomene in der Sprache der Wirtschaft zu neuen Wortbildungsprodukten führen und wie sie zur Entwicklung der Wirtschaftssprache beitragen. Diese
\end{abstract}

\begin{abstract}
Entwicklung wirft wiederum neue Herausforderungen an die Fremdsprachendidaktik auf, da Fachphrasen aus verschiedenen Wirtschaftsbereichen zunehmend in die Alltagssprache eindringen.
\end{abstract}

Schlüsselwörter: Wirtschaftssprache, Wirtschaftspresse, verbale Phrase, Metapher

\section{EINLEITUNG}

Der Beitrag ist die Fortsetzung einer erfolgten längeren Analyse zum Thema „Phrasen in der Wirtschaftssprache“ , die einige Gedanken zum Gebrauch beliebter Phrasen der Wirtschaftspresse des ausgehenden 19. Jahrhunderts im Vergleich zum ausgehenden 20. Jahrhundert darstellt. Anhand des erfolgten Vergleichs wurde die Entwicklung der Wirtschaftssprache in diesem Zeitraum gezeigt. Im Folgenden soll ein Ausblick ins 21. Jahrhundert gewagt und Wortbildungsphänomene in der Sprache der 
Wirtschaft gezeigt werden, die zu neuen, nicht immer auf Anhieb verständlichen Wortbildungsprodukten führen. Das Wissen um diese spezifischen Redensarten kann beim Erschließen von Wirtschaftstexten und beim Erlernen der Fachausdrücke der Wirtschaftssprache von Nutzen sein, sei es für den angehenden Germanisten, sei es für den Deutsch lernenden Wirtschaftswissenschaftler, sei es auch für den Laien, da Fachphrasen aus verschiedenen Wirtschaftsbereichen zunehmend in die Alltagssprache eindringen. Damit wird die Frage aufgeworfen, welche neuen Herausforderungen diese Entwicklung an die Fremdsprachendidaktik stellt.

\section{UNTERSUCHUNGSGEGENSTAND UND QUELLEN}

Die Auswahl von Beispielen wird in Satzkontexten aus Presseartikeln des ausgehenden 20. Jahrhunderts gezeigt. Mit Hilfe von mehreren Zitaten werden Ausdrücke der Wirtschaftssprache analysiert und anhand von Wirtschaftswörterbüchern erläutert. Untersuchungsgegenstand ist der Wortschatz aus folgenden Wirtschaftszeitschriften: „Wirtschaftskurier. Nachrichten und Kommentare aus dem Industrie- und Finanzbereich“ 1995 und 1996 (WK), „Die Wirtschaft. Unternehmen-Märkte-und Unternehmensführung" mehrere Ausgaben von 1995 (DW und „Deutsche Handwerkszeitung“ 1993 (DHZ). Dem Nachschlagen der Wörter und Phrasen dienten zwei Wirtschaftswörterbücher von Gabler und Vahlens, Ausgaben von 1996. Der in den Wörterbüchern enthaltene Wortschatz ist jedoch nicht der Gegenstand der Analyse. In der Untersuchung ging es um die Frage, welche Phrasen in der Wirtschaftssprache häufig sind, welche für den Augenblick geschaffen wurden, und schließlich wie hermetisch die neuen sprachlichen Phänomene für den Leser der Wirtschaftspresse sein können.

\section{FACHSPRACHE UND ALLGEMEINSPRACHE}

Der technische Fortschritt im 19. und 20. Jahrhundert, die rege wirtschaftliche Entwicklung, die Entwicklung des Handels und der Industrie sowie das Aufgehen der traditionellen gesellschaftlichen Strukturen sowie Veränderungen in der Arbeitswelt hatten einen starken Ausbau des Wortschatzes zur Folge. Neben etablierten Phrasen, die von all den Erfindungen und Umwälzungen unberührt bleiben, werden stets neue Phrasen "generiert". Fachsprachenforscher sprechen von der „abnehmenden Verwendungsdauer" der Bedeutungen, an der sich die Entwicklungsgeschwindigkeit in den Fachbereichen messen lasse [Roelcke 1999] und davon, dass der Fachwortschatz immer schneller „umgeschlagen“ wird und daher schneller veraltet. Die Fachsprachen und so auch die Wirtschaftssprache, sind auch in immer stärkerem Maße „internationalisiert, komprimiert und kompliziert" [Fluck: 1998]. Das ist besonders deutlich in der Wirtschaftspresse zu sehen. Wir können daher über von Wirtschaftsjournalisten verwendete „Fachphrasen“ sprechen. Diese werden von der Wirtschaftspresse und den modernen Medien weiter getragen und haben einen zunehmenden Einfluss auf die Allgemeinsprache. Fachsprachenforscher sprachen bereits Ende des 20. Jahrhunderts von einer "Verfachsprachlichung der Standardsprache" [Roelcke 1999]. Diese Erscheinung lässt sich auch im 21. Jahrhundert weiter beobachten. Die in die Wirtschaftstexte eingebauten Phrasen sind aber für den Leser immer weniger verständlich. Daher ist das Interesse der Forschung an der Entwicklung der Sprache der Wirtschaft und der Abklärung häufig benutzter Fachphrasen sehr willkommen, denn es schafft „Voraussetzungen für die Kommunikation zwischen Fachleuten und Laien" [Fluck 1998]. 


\section{WORTSCHATZBEISPIELE}

4.1. Auswahl von $\begin{aligned} & \text { Phrasen } \\ & \text { zusammengesetzten } \\ & \text { Partizipien }\end{aligned}$

mit und

Eine Gruppe, die sich sehr dynamisch entwickelt, sind die in der Wirtschaftspresse auftretenden zusammengesetzten Adjektive und Partizipien in Fügungen mit Substantiven. Für die vorliegende Analyse konnten etwa 60 solcher Wörter gefunden und ausgewertet werden. Einige werden im Folgenden präsentiert. Die erste Gruppe umfasst native Adjektive und Partizipien in Phrasen mit einem nativen bzw. nichtnativen Verb; in der zweiten Gruppe sind in ähnlichen Phrasen nichtnative Adjektive und Partizipien zusammengefasst. Innerhalb der Gruppen treten die Beispiele in alphabetischer Reihenfolge auf.

Gruppe 1: Native Wörter in Phrasen

ertragsstark, volumenstark, umsatzstark, leistungsstark $\rightarrow$ ertragsstarker Herbst, volumenstärkster Bereich, umsatzstarke Monate, umsatzstärkstes Unternehmen, umsatzkräftigster Markt

"Der normalerweise besonders ertragsstarke Herbst ist im vergangenen Jahr ungewöhnlichen Schwankungen unterlegen.", WK 38/Januar 1996, S.16

Es drückt präzise aus, dass für ein Unternehmen in einer Zeitspanne die Erträge besonders hoch sind.

"SNI habe sich im letzten Geschäftsjahr zum volumenstärksten Siemens-Bereich entwickelt (...)"

WK 38/Januar 1996, S.14

Damit kann u.a. ausgedrückt werden, dass in einem Bereich mengenmäßig sehr viel hergestellt wird

"Da die zwei umsatzstarken Monate November und Dezember noch nicht eingerechnet sind (...)"

WK 38/Januar 1996, S.20.
"(...) die als umsatzstärkstes Unternehmen der Gruppe mit ihrem Beitragsplus den Marktschnitt wieder übertreffen konnte."WK 38/Januar 1996, S.12

In einem Zeitraum wurde besonders viel verkauft.

"Im Visier hat Escom neben dem umsatzkräftigen PC-Markt Großbritannien auch Skandinavien und die Schweiz." WK 38/Januar 1996, S.13

Es ist ein Synonym zu umsatzstark.

"Sie sind beim XM leistungsstark und elastisch in den entscheidenden Drehzahlbereichen." WK 38/Januar 1996, S.24

Es ist auch aus anderen Lebensbereichen bekannt (leistungsstarke Schüler).

flächendeckend -> flächendeckende Aktivitäten, Akquisitionen, Anwesenheit

"Vor allem durch Akquisitionen soll zudem die Landspedition künftig in Europa flächendeckend präsent werden." WK 38/Januar 1996, S.15".

Das Unternehmen versucht den ganzen Markt zu decken, um die Konkurrenz auszuschalten.

führend, technologisch führend $\rightarrow$ auf dem Markt führend ? marktführend

"Mit einem Geschäftsvolumen von rund 6000 Millionen DM ist das Geschäftsgebiet (...) am Weltmarkt führend." WK 38/Februar 1996, S.15 "Wir werden unser Wohlstandsniveau nur halten können, wenn es uns gelingt, technologisch führend $z u$ bleiben." WK 38/Januar 1996, S.1

Der Ausdruck bezeichnet den ersten, bzw. einen der ersten Plätze auf dem Markt.

gesamtwirtschaftlich-> gesamtwirtschaftliche Lage, Situation

"(...) vor dem Hintergrund der gesamtwirtschaftlichen Situation und im Branchenvergleich kann jedoch die Umsatzentwicklung durchaus befriedigen." WK 38/Januar 1996, S.12.

Dieses Adjektiv dient der Bezeichnung der Lage in allen Wirtschaftszweigen. 
gesättigt $\rightarrow$ gesättigte und ungesättigte Märkte

"Gerade in Märkten haben Unternehmen, wenn sie Fehler machen, nur zwei Möglichkeiten (...)." WK 37/Mai 1995, S.2

Es ist ein altbewährter Begriff aus der Volkswirtschaftslehre, der fest etabliert ist.

kostengünstig, kostensparend, preisgünstig, -> kostensparende Zerlegung, preisgünstigste Stadt, preisgünstiges Angebot

"Bei perfekter Organisation können schriftliche und telefonische Betreuung in gleicher Qualität schneller und kostengünstiger als durch Vertreter erfolgen. "WK 38/Januar 1996, S.11

"Der recyclingfreundliche modulare Aufbau, der eine schnelle kostensparende Zerlegung des Altgeräts erlaubt (...)" WK 37/Mai 1995, S. 21 "Preisgünstigste Stadt im oberen Bereich ist Haldensleben (...)" DW 18/4.Mai 1995, S.21

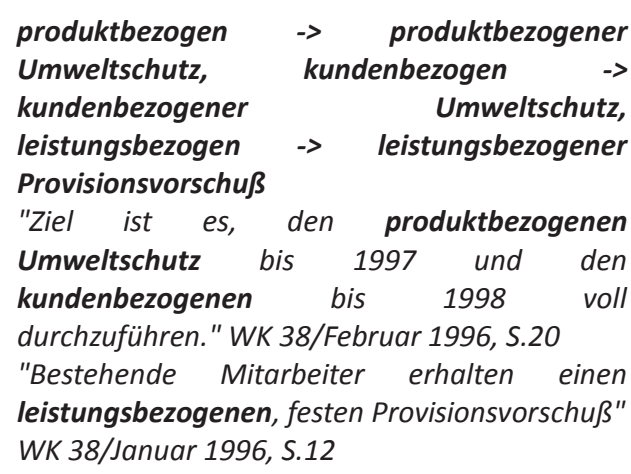

leistungsorientiert $\quad \rightarrow \quad$ leistungsorientierte Vergütung, konsumorientiert $\quad \rightarrow$ konsumorientierte Einkommensbesteuerung "Unter Berücksichtigung der(...) Stärken wie leistungsorientierte Vergütung (...) (...)" WK 38/Januar 1996, S.12 Der Lohn bzw. das Gehalt hängt von der Leistung ab.

"Nach dem Konzept einer konsumorientierten und damit auch marktverträglichen Einkommensbesteuerung ist der Sparerfreibeitrag ein (...) Ansatz auf dem Weg zu einer systematischen Lösung. "WK 38/Januar 1996, S.3 marktgängig, marktgerecht, marktverträglich > marktgerechte Ausrichtung, marktgängige Papiere, marktverträgliche Einkommensbesteuerung

In die Reihe beliebter Neuprägungen der Wirtschaftssprache gesellen sich Zusammensetzungen mit dem Bestimmungswort Markt-:

"Ein Papier ist nur dann marktgängig, wenn eine tägliche Kursnotierung zustande kommt." WK 37/Mai 1995, S.3

"Die marktgerechte Ausrichtung des Geschäftsfeldes Fassade wird durch die Suche nach strategischen Partnern energisch vorangetrieben." WK 38/Januar 1996, S.16

wettbewerblich, konkurrenzfähig $\rightarrow$ wettbewerbliche Marktstrukturen

"Die Kooperation stellt einen wesentlichen Beitrag zur Entwicklung wettbewerblicher Marktstrukturen in Deutschland dar." WK 38/Februar 1996, S.16

Es sind zwei Synonyme, wobei das native Wort immer mehr von dem nichtnativen verdrängt wird.

wirtschaftspolitisch -> wirtschaftspolitische Aspekte, wirtschaftspolitische Sprecherin, wirtschaftspolitische Agenda

In den Bereich Wirtschaft und Politik gehört die Zusammensetzung „wirtschaftspolitisch”, die von den gegenseitigen Bezügen der beiden Bereiche zeugt.

"Wir müssen gerade unter wirtschaftspolitischen Aspekten einige Wahrheiten sehen (...)" WK 38/Januar 1996, S.1

"Margareta Wolf ist nicht nur wirtschaftspolitische Sprecherin der BündnisGrünen im Deutschen Bundestag; sie hat auch eine spitze Zunge. "WK 38/Januar 1996, S.1

"Ganz oben auf der wirtschaftspolitischen Agenda steht (..)eine Unternehmenssteuerreform (...)"

WK 38/Januar 1996, S.2

zielgruppengerecht -> zielgruppengerechtes Mail-Order-Geschäft 
Immer häufiger sind zusammengesetzte Adjektive und Partizipien anzutreffen, die mehr als zwei Bestandteile zählen. Auch sie sollen der Tendenz Rechnung tragen, dass die Wirtschaftssprache möglichst kurze und präzise Ausdrücke hervorbringen soll.

"Durch die Kombination (...) schafft sich Escom die Basis für ein zielgruppengerechtes und damit intelligentes Mail-Order-Geschäft" WK 38/Januar 1996, S.13

Hier kommt zum Ausdruck, dass etwas im Hinblick auf die anzusprechende Zielgruppe (also die Gesamtheit aller potentiellen Personen, die mit einer bestimmten Marketingaktivität angesprochen werden sollen) gemacht wird.

\section{Gruppe 2: Nichtnative Wörter in Phrasen} boomend $\rightarrow$ boomender Markt, boomender Baumarkt, boomende Autotochter

"Angreifen will Porsche außerdem im derzeit boomenden Markt der Roadster (...)"

WK 38/Januar 1996, S.14 "Fertig zur Fahrt auf der Überholspur"

"Immer mehr Unternehmen (...) drängen auf den boomenden Baumarkt in Deutschland."

WK 37/Mai 1995, S.20 "Lokomotive noch unter Dampf?"

"Doch selbst bei der boomenden Autotochter Mercedes-Benz wird mehr Rotstift angesetzt (...)"

WK 37/Mai 1995, S.17 "70 000 Arbeitsplätze gestrichen - Stern aufpoliert"

Das Partizip wird abgeleitet vom Substantiv "Boom" (Aufschwung, wobei das Substantiv selbst von dem Fremdwort nicht verdrängt ist).

extern, intern $\rightarrow$ externe Kunden, interne Umbesetzungen, firmenintern $\rightarrow$ firmeninterner Wettstreit

"Alle Geschäftsbereiche (...) wickeln mehr als die Hälfte ihres Umsatzes mit externen Kunden $a b . "$

WK 38/Februar 1996, S.17

"Die verbleibenden (...) Stellen sollen (...) durch natürliche Fluktuation, interne Umbesetzungen oder Vorruhestandregelungen abgebaut werden."
WK 38/Februar 1996, S.16. Damit ist ein Stellenwechsel innerhalb des Unternehmens:

"Im firmeninternen Wettstreit wollen wir im ersten Drittel bleiben."WK 38/Februar 1996, S.15

Extern sind Kunden, die außerhalb des Unternehmens zu suchen sind. Diese Fremdwörter sind auch in der Allgemeinsprache bekannt, denn sie ersetzen die Wörter außerhalb und innerhalb, z.B. das Wort firmenintern umschließt alles, was sich innerhalb der Firma abspielt. Typisch für die Unternehmenskultur sind z.B. firmeninterne Rundschreiben.

\section{forciert -> forciertes Wachstum}

"Mehr Ausgewogenheit strebe das Unternehmen (...) durch forciertes Wachstum im Ausland an."

WK 38/Februar 1996, S.15 "12000 Neueinstellungen: Lichtblicke für den Standort Deutschland"

Das Adjektiv wird von dem Verb forcieren in der Bedeutung von vorantreiben, steigern, beschleunigen, aber auch erzwingen, abgeleitet.

geleast, verleast $\rightarrow$ verleaste Fahrzeuge, verleaste Anlagen

Zwei andere beliebte Partizipien sind auf das Verb leasen zurückzuführen. Es bedeutet mieten oder pachten, wird aber in der Wirtschaft auf das Leasen von Investitionsgütern oder Industrieanlagen bezogen:

"(...) im vergangenen Jahr haben diese Banken 646000 Wagen finanziert oder verleast."

"25\% der verleasten Fahrzeuge gingen an Private (...) Wirtschaft." WK 37/Mai 1995, S.10

"Ende 1994 (...) addierte sich der Bestand der verleasten Anlagen auf einen Wert von 228 Mrd. DM (...)" WK 38/Januar 1996, S.15

innovativ, kreativ, international -> innovative Motorentechnik, Dienstleistungen, internationales Geschäft

Es handelt sich um Adjektive, die in der Allgemeinsprache bekannt sind: innovativ ist aus der Technik übernommen, kreativ aus der 
Kunst, international ist allgemeinsprachlich. Hier die wirtschaftlichen Kontexte :

"Ein Fahrzeug (...) mit einem luxuriösen Innenraum und nicht zuletzt mit einer innovativen Fahrwerks- und Motorentechnik (...)" WK 38/Januar 1996, S.24

"Technologische Veränderungen (...) fordern den Großhandel, sich schnell innovativ und kreativ anzupassen (...)"DW 18/4.Mai 1995, S.12

"Mannesman (...) und Vebacom werden (...) zur Entwicklung innovativer Dienstleistungen beitragen."

WK 38/Februar 1996, S.16

"Durch die Aufwertung in DM wurde aus dem Plus im internationalen Geschäft ein Zuwachs (...)"

WK 38/Februar 1996, S.16

"Der Wandel vom deutsch denkenden zum international orientierten Unternehmen sei voll in Gang gekommen." WK 38/Februar 1996, S.16

informationstechnisch, großvolumig $\rightarrow$ informationstechnische Dienstleistungen, großvolumige Brennstoffsparte

Hier zwei typische mehrgliedrige Adjektive, die der Sprachökonomie Rechnung tragen:

"Als größter unabhängiger Anbieter von informationstechnischen Dienstleistungen in Europa (...) werden Debis und Cap Gemini Sogeti diese Position (...) stärken." WK 38/Februar 1996, S.17

"Die früher mit 4 Mrd. DM Umsatz großvolumige Brennstoffsparte wurde weiter zurückgefahren."

WK 38/Februar 1996, S.18

konjunkturbedingt $\rightarrow$ konjunkturbedingter Ergebnisrückgang

Mehrere Adjektive sind auf das Substantiv „Konjunktur” zurückzuführen.

"Das Geschäftsfeld Fenster und Türen ist in besonderem Maße von einem konjunkturbedingten Ergebnisrückgang betroffen. "WK 38/Januar 1996, S.16

konjunkturell -> konjunkturelle Schwächen, Besserung, Belebung, Einflüsse
Ein Schlagwort ist das Adjektiv "konjunkturell”, hier in typischen Phrasen:

"Erste konjunkturelle Schwächen zeichnen sich allerdings (...) bei der stagnierenden Preisentwicklung ab." DHZ 45/3. Dez. 1993, S.12 "(...) die politischen Rahmenbedingungen seien alles andere als geeignet für eine schnelle konjunkturelle Besserung" DHZ 45/3. Dez. 1993, S.12

"Bei Packpapier (...) setzte die konjunkturelle Belebung (...) im Laufe des Jahres 1994 ein (...)" DW 18/4. Mai 1995, S.12

"Im Geschäftsfeld Fassade machten sich die konjunkturellen Einflüsse am stärksten bemerkbar."

WK 38/Januar 1996, S.16

strukturell -> strukturelle Arbeitslosigkeit

Ein ähnlich gebildetes Adjektiv ist das von dem Substantiv Struktur (Wirtschaftsstruktur) abgeleitete Wort strukturell, das besonders häufig in der Verbindung strukturelle Arbeitslosigkeit anzutreffen ist:

"Die strukturelle Arbeitslosigkeit wird leider hoch bleiben (...)" WK 38/Januar 1996, S.2

konsolidiert $\rightarrow$ konsolidierter Umsatz, konsolidierte Umsätze

Hier handelt es sich um einen Fachbegriff; Die Konsolidierung ist nach Vahlens ein „spezieller Vorgang bei dem die bezüglich einer bestimmten ökonomischen Aktivität gleichartigen Transaktionen zwischen Wirtschaftseinheiten eines Sektors oder der Volkswirtschaft gegeneinander aufgerechnet werden".

"(...) erzielte 1994 mit rund 1900 Mitarbeitern einen konsolidierten Weltumsatz von 84000 Mio. DM." WK 38/Januar 1996, S.20

"Wenn das Geschäftsjahr 1995 abgerechnet sein wird, dürfte ein konsolidierter Konzernumsatz von rund 530 Mio. DM erreicht worden sein." WK 38/Januar 1996, S.20

"Einschließlich der nicht konsolidierten Umsätze ergebe sich ein Umsatz (...) unter 3 Mrd. DM."

WK38/Februar 1996, S.18 
kundenorientiert -> kundenorientierter Service, kundenorientierte Beratung

Hier eine Ergänzung zu Partizipien mit dem Wort -orientiert: Es wird betont, dass die Aktivitäten der Firma auf den Kunden zielen und er zuvorkommend behandelt wird.

"Unter dem Stichwort kundenorientierter Service versuchen die Gesellschaften, die bestehenden Geschäftsfelder auszubauen (...)" WK 37/Mai 1995, S.10

"Sie stellen die kundenorientierte Beratung vor Ort sicher (...)"WK 38/Januar 1996, S.11

\section{vertriebsorientiert}

Aus der immer größer werdenden Gruppe der Zusammensetzungen mit dem Wort ,-orientiert" sei an dieser Stelle noch das Partizip „vertriebsorientiert" zitiert:

"Das erste ist stark vertriebsorientiert, das zweite beruht vor allem auf individuell zugeschnittenen Finanzdienstleistungen." WK 38/Januar 1996, S.10

profitabel, rentabel -> rentable, unrentable Unternehmen

"Die Debis AG arbeitet in allen Geschäftsbereichen profitabel und erfolgreich." WK 38/Februar 1996, S.17

"Andererseits mußten auch Rückstellungen für Betriebsschließungen und den Austritt aus einigen unrentablen Unternehmen gebildet werden. "WK38/Februar 1996, S.18

stabilisiert, privatisiert $\quad->\quad$ stabilisierte Konjunktur, privatisierte Betriebe

"(...) überall hinterlassen die Fachleute des Ende 1994 zum zweiten Mal privatisierten Baubetriebes ihre Spuren." DW 18/4. Mai 1995, S.8

"Im Geschäftsjahr 1994/95 (...) schwächten sich bei den Gesellschaften der Thyssen IndustrieGruppe und stabilisierter Weltkonjunktur die Wachstumsraten leicht ab." WK 38/Februar 1996, S.18

steigend, stagnierend -> steigende, stagnierende Tendenz

Die Partizipien bieten eine vorsichtige Einschätzung der Wirtschaftslage:
"Von den 44 Lebensmittelmärkten (...) wiesen 29 eine rückläufige Tendenz, 2 eine stagnierende und nur 13 eine steigende Tendenz aus." WK38/Februar 1996, S.18

strategisch -> strategische Produktgruppe, strategische Ausrichtung

Das aus der Militärsprache übernommene Adjektiv hat sich in Wirtschaft durchgesetzt. Substantive wie Wirtschaftsstrategie sind in die Allgemeinsprache eingegangen.

"Raeber zeigte sich überzeugt, die neue Beteiligung in absehbarer Zeit aufstocken zu können, um dann diese strategische Produktgruppe weiter auszubauen."

"In der strategischen Ausrichtung wolle man die Marktorientierung steigern (...)" WK 38/Februar 1996, S.16

synergetisch -> synergetische Effekte

Ein Schlagwort ist in der Wirtschaftspresse das Substantiv Synergie, davon abgeleitet das Adjektiv synergetisch. Der synergetische Effekt (Synergieeffekt) ist ein Phänomen, nach dem eine Gesamtheit größere Erfolge verzeichnet als die Summe der dazugehörigen Teile (Vahlens)

"Von Jahresmitte 1995 an bis Ende 1997 werden zur Gewinnung synergetischer Effekte erst einmal insgesamt ca. 47 Mio. DM an Strukturkosten anfallen." WK 37/Mai 1995, S.12

\subsection{Auswahl von Metaphern}

Entgegen dem seriösen Erscheinungsbild mancher Fachzeitschriften erweisen sich die Metaphern als ein beliebtes Ausdrucksmittel der Publizistik. Sie lockern den berichtenden Stil auf und akzentuieren inn auf bildhafte Weise (Reger:1980:80). Sie sind ein Stilmerkmal, das der Abstraktion in der Öffentlichkeitssprache entgegenwirkt und lesemotivierend wirkt [Reger 1980: 95]. Metaphern sind sprachliche Bilder, die auf einer Ähnlichkeitsbeziehung zwischen zwei Gegenständen bzw. Begriffen beruhen. Aufgrund gleicher oder ähnlicher Bedeutungsmerkmale findet eine Bedeutungsübertragung statt. Diese Definition sei hier nach Bußmann [1990: 484] zitiert, es sollen jedoch nicht alle Typen von Metaphern analysiert werden. Es werden hier 
hauptsächlich die "dynamisierende Metapher" (in der einer dinghaften Erscheinung Bewegung oder mehr Bewegung verliehen wird, z.B.: „Der Geschäftsführer steigt hart ein") , die „personifizierende Metapher" (in der einem Gegenstand oder einer Erscheinung menschliche Eigenschaften und Gefühle zugeschrieben werden, z.B.: „Die Bauindustrie war enttäuscht") sowie die "Metapher als Bild" (in der mit bildhafter Ausdrucksweise eine Erscheinung beschrieben wird) auftreten.

Man unterscheidet auch den Grad der Lexikalisierung der Metapher (verblasste Metapher, konventionelle Metapher, okkasionelle Metapher) sowie den Grad ihrer Komplexität (eingliedrige und mehrgliedrige Metapher). Viele Phrasen stehen an der Grenze zum metaphorischen Gebrauch. Diese Grenzen sind sehr fließend, denn die Tatsache, ob ein Ausdruck als Metapher gewertet wird, hängt vom Satzkontext, letztendlich aber auch vor der Einstellung des Lesers ab. Metaphern haben mehrere Auslegungsmöglichkeiten, ihr Verständnis setzt „verstehenswillige Empfänger" voraus (Kroeber-Riel:1969:80). Die in der Presse gebrauchten Metaphern sind zumeist "konjunkturabhängig", da sie die Wirtschaftslage widerspiegeln.

\section{Beispiele:}

In der Sprache des 20. Jahrhunderts gibt es zahlreiche eingliedrige und mehrgliedrige Metaphern mit dem Wort Markt. Viele reihen sich in die thematische Gruppe ein, die sich von Analogien zu bestimmten Verhaltensweisen beim Spiel oder im Kampf speisen [Kroeber-Riel 1969: 84]. ${ }^{3}$

\section{Marktführer, Marktleader}

Es ist eine der häufigsten personifizierenden Metaphern. Gemeint ist kein Mensch, sondern ein auf dem Markt führendes Unternehmen:

"Disetronic ist eine (...) wenig bekannte Firma, dies, obwohl sie in der Medizinaltechnik zu den Marktführern weltweit gehört." WK 38/Januar 1996, S.19 "Marktleader in der Medizinaltechnik vor dem Gang an die Börse"

${ }^{3}$ Kroeber-Riel 1969, S. 84.

\section{Marktszenario}

Es handelt sich hier um eine Metapher des „okkasionellen" Typs, die theatralisch anmutet:

"Die Risiken für dieses optimistische Marktszenario sind (...) auch in Deutschland zu sehen."

WK 38/Januar 1996, S.2 "Konjunktur- und Zinsprognose 1996 (...)"

\section{Marktanteile -> um Marktanteile kämpfen}

Die Metapher, die sich auf Analogien zum Kampf stützt, hat sich aufgrund des häufig gebrauchten Modewortes Marktanteil etabliert: "In diesem Umfeld kämpft Jean Pascale um Marktanteile." WK 38/Januar 1996, S.20

\section{Markt -> etwas auf den Markt bringen}

Diese kaum noch als Metapher gewertete Wendung basiert auf dem Bild der konkreten Situation, in der der Hersteller seine Ware zum Verkauf auf den Markt bringt. Heute geht es um neue Produkte:

"Wie lange dauerte es, bis Sie Ihr erstes Gerät auf den Markt bringen konnten?" WK 38/Jan.1996, S.19

Märkte -> Märkte aufbauen, ausbauen, erobern, sichern

Auch die Fügung Märkte erobern stützt sich auf die Analogie zu den im Kriege eroberten Gebieten. Märkte aufbauen und Märkte ausbauen rufen dagegen Assoziationen zum Häuserbau hervor:

"Unter dem Zeichen 'Ecomade in Germany' sollen deutsche Unternehmen die Märkte für Umwelttechnik (...) und für Umweltdienstleistungen sichern, ausbauen oder erobern." DW 18/4.Mai 1995

"(..) um (...) chancenreich die Märkte der Zukunft mit aufzubauen." DHZ 23/3.Dez.93, S.3

\section{Exporte vorantreiben}

Bei diesem Metaphernbild hat man die Assoziation mit einem Hirten, der seine Tiere vorantreibt, damit sie schneller gehen:

"In den aufstrebenden Nationen drängen die Kräfte des freien Marktes die Regierungen, (...) 
die Exporte voranzutreiben". WK 38/Februar 1996, S.5

\section{Arbeitsplätze exportieren}

Hier handelt es sich um die Schaffung von Arbeitsplätzen in ausländischen Niederlassungen des Unternehmens im Falle einer Verlagerung der Produktion.

"Infolge der geringeren Kosten werden viele der deutschen Arbeitsplätze in diese Regionen exportiert." WK 38/Januar 1996, S.2

\section{in die Wachstumsregionen vorstoßen}

Diese okkasionelle Fügung mit einem Verb aus der Sprache des Militärs ist eine klassische Metapher des Kampfes:

"Deshalb müssen unsere Unternehmen in die Wachstumsregionen der Welt vorstoßen." WK 38/Feb.1996, S.3

4.3. Fügungen von Substantiv und Verb, verbale Phrasen

Es sollen noch einige Wortbildungsprodukte genannt werden, die aus einem Substantiv und einem Verb bestehend, usuelle verbale Kollokationen und Funktionsverbgefüge bilden. Der Kontext wird an dieser Stelle ausgespart , da diese Fügungen die zeitgenössischen Wirtschaftspresse sehr zahlreich bevölkern. Manche von ihnen sind zu festen Wendungen geworden, viele werden so oft verwendet, dass sie zu Schlagwörtern der Wirtschaftssprache geworden sind. Dazu gehören u.a. Wendungen mit Verben, die Wirtschaftswachstum signalisieren, wie z.B. das Verb steigern oder Verben mit positivem Klang wie verbessern, optimieren, erwirtschaften, gewinnen, erzielen, ausbauen, ausweiten oder entwickeln. Hier einige Bespiele von häufig auftretenden Fügungen (in der alphabetischen Reihenfolge der Substantive): ein Angebot unterbreiten, das Ergebnis verbessern, den Ertrag steigern, die Erträge optimieren, Führungsaufgaben delegieren, neue Geschäftsfelder erschließen, Gewinne erwirtschaften, einen Gewinn erzielen, die Kapazitäten verlagern, auf den Markt zielen, im Markt agieren, Marktanteile gewinnen, die führende Position ausbauen, eine
Produktgruppe ausbauen, Produkte entwickeln, Produkte und Technologien entwickeln, die Produktionstechnik transferieren, Risiken streuen, den Umsatz steigern, das Service- und Dienstleistungsangebot ausbauen, den Umsatz ausweiten, einen Umsatz erwirtschaften. Diese Auflistung spiegelt nicht nur eine Reihe von wichtigen Tätigkeiten im modernen Wirtschaftsleben wider, sie liefert auch Hinweise zu der Thematik der Wirtschaftstexte sowie Stoff für eventuelle Vokabellisten. Mit Blick ins 21. Jahrhundert, kann aufgrund der durchgeführten Untersuchung die Prognose aufgestellt werden, dass immer mehr komplizierte Phrasen und metaphorische Ausdrücke in die Wirtschaftssprache eingehen werden und später oder gleichzeitig in die Allgemeinsprache übergleiten. Sie werden von Lesern, die entweder über zu schwache Fremdsprachenkenntnisse oder aber über zu schwache Wirtschaftskenntnisse verfügen, oftmals beim Lesen übersprungen, da ihr Sinn schwer zu verstehen ist.

\section{FACHSPRACHENDIDAKTIK IM 21. JAHRHUNDERT}

Die vorgeführten Beispiele stellen immer neue Herausforderungen an die Didaktik der Fremdsprache Wirtschaftsdeutsch. Vor diese Herausforderungen wird der Lehrbeauftragte gestellt, wenn er im Wirtschaftsdeutschuntericht mit authentischen Wirtschaftstexten arbeiten will. Muss man literarisch bewandert sein, um die Metaphern der Wirtschaftssprache richtig zu verstehen? Müssen Wirtschaftswissenschaftler Wortbildungsmuster kennen, um die Phrasen der Wirtschaft richtig zu verstehen? Im Wirtschaftsdeutschunterricht an der Philologie werden von den Studenten andere Fähigkeiten und Fertigkeiten als an einer Wirtschaftshochschule verlangt, auch ist der Ausgangspunkt ein anderer, da die Studenten über keine volks- oder betriebswirtschaftlichen Kenntnisse verfügen. So ist es die Rolle des Dozenten, nicht nur die Sprache zu unterrichten, sondern auch den Prozess der Aneignung des wirtschaftlichen Wissens zu 
Wie soll der Fachsprachenunterricht im 21. Jahrhundert sein?

fördern. Germanistikstudenten verfügen über gute bis sehr gute Deutschkenntnisse, man Auseinandersetzung mit wirtschaftlichen Begriffen von großem Nutzen sein. Es können auch gezielt Übungen zur Wortbildung kann aber nicht an ihre wirtschaftlichen Erfahrungen anknüpfen. Das Wirtschaftswissen muss ihnen neben der Sprache mitvermittelt werden oder sie müssen es sich selbstständig eingesetzt sowie eine kritische Diskussion über die Wortbildungstendenzen der aneignen. Hierbei kann eine semantische

\section{BIBLIOGRAPHIE:}

[1] Fluck, H.-R. (1998): Fachsprachen und Fachkommunikation. Heidelberg. (Studienbibliographien Sprachwissenschaft; 26)

[2] Kroeber-Riel, W. (1969): Wissenschaftstheoretische Sprachkritik in der Betriebswirtschaftlehre. Semantische und pragmatische Untersuchungen betriebswirtschaftlicher Sprachen. Berlin: Duncker\&Humblot.

[3] Reger, H. (1980): Metaphern und Idiome in szenischen Texten, in der Werbe- und Pressesprache. Hamburg: Buske.

[4] Roelcke, Th. (1999): Fachsprachen. Berlin.

\section{WÖRTERBÜCHER:}

[5] Bußmann, H. (1990): Lexikon der Sprachwissenschaft. Stuttgart.

[6] Gabler Wirtschaftslexikon in 8 Bänden (1993). Wiesbaden.

[7] Vahlens Großes Wirtschaftslexikon in 4 Bänden (1994). München. 